\title{
FATORES DIFICULTADORES NA REALIZAÇÃO DAS TECNOLOGIAS LEVES NO CUIDADO DO ENFERMEIRO NA ATENÇÃO BÁSICA
}

\section{DIFFICULTY FACTORS IN CARRYING OUT THE LIGHT TECHNOLOGIES IN THE CARE OF THE NURSE IN BASIC ATTENTION}

\section{FACTORES DIFICULTADES EN LA REALIZACIÓN DE LAS TECNOLOGÍAS LEVES EN EL CUIDADO DEL ENFERMERO EN LA ATENCIÓN BÁSICA}

Joyce Wadna Rodrigues de Souza ${ }^{1}$, Fabrícia Cristina Vidal Silva ${ }^{2}$, Paloma Karen Holanda Brito ${ }^{3}$, Rayara Cibelle Ribeiro da Silva ${ }^{4}$, Bruna Alves ${ }^{5}$, Marcelo Costa Fernandes ${ }^{6}$

\begin{abstract}
RESUMO
Objetivo: identificar os obstáculos encontrados na execução de atividades relacionadas às tecnologias leves voltadas ao cuidado do enfermeiro na Atenção Básica. Método: Trata-se de um estudo descritivo com abordagem qualitativa realizado com 15 Enfermeiros das Estratégias de Saúde da Família do município de Cajazeiras, Paraíba. Foi utilizado para coleta de dados uma entrevista semiestruturada, que aconteceu entre os meses de janeiro e fevereiro de 2016, após a aprovação do Comitê de Ética e Pesquisa. Resultados: Percebeu-se a existência de alguns fatores que prejudicam a utilização das tecnologias leves do cuidado, dentre eles, o tempo reduzido para realizar o atendimento à população, estrutura física inadequada, imediatismo do atendimento, a pressão da demanda para o trabalho. Conclusão: É imprescindível que exista o apoio da gestão oferecendo ações de educação permanente para com os profissionais da saúde sobre a importância da utilização das tecnologias interacionistas em seus cotidianos de práticas.
\end{abstract}

Palavras-chave: Cuidados de Enfermagem. Enfermagem. Atenção primária à saúde.

\begin{abstract}
Objective: to identify the obstacles encountered in the implementation of activities related to light technologies aimed at the care of nurses in Primary Care. Method: This is a descriptive study with a qualitative approach carried out with 15 Nurses of the Family Health Strategies of the municipality of Cajazeiras, Paraíba. A semistructured interview was used for data collection, which took place between January and February 2016, after approval by the Ethics and Research Committee. Results: The existence of some factors that hinder the use of light care technologies, among them, reduced time to attend to the population, inadequate physical structure, immediacy of care, and the pressure of demand for work were noticed. Conclusion: It is essential that there is support from the management offering ongoing education actions with health professionals about the importance of the use of interactionist technologies in their everyday practice.
\end{abstract}

Keywords: Nursing Care. Nursing. Primary Health Care.

\footnotetext{
${ }^{1}$ Enfermeira, UFCG, Cajazeiras, PB - Brasil. Email: wadnajoyce@gmail.com

2 Enfermeira, UFCG, Cajazeiras, PB - Brasil. Email: fabrícia.vidal23@gmail.com

${ }^{3}$ Enfermeira, UFCG, Cajazeiras, PB - Brasil. Email: pah.karen@ hotmail.com

${ }^{4}$ Enfermeira, UFCG, Cajazeiras, PB - Brasil. Email: rayararibeiro@ outlook.com

${ }^{5}$ Acadêmica de enfermagem, UFCG, Cajazeiras, PB - Brasil. Email: brunaalves0117@gmail.com

${ }^{6}$ Enfermeiro. Doutor pelo Programa de Pós-graduação Cuidados Clínicos em Enfermagem e Saúde pela UECE. Líder do Grupo de Pesquisa Laboratório de Tecnologias de Informação e Comunicação em Saúde - LATICS/ UFCG/ CNPq, Cajazeiras, PB - Brasil. Email: celo_cf@ hotmail.com.
} 


\section{RESUMEN}

Objetivo: identificar los obstáculos encontrados en la ejecución de actividades relacionadas con las tecnologías ligeras dirigidas al cuidado del enfermero en la Atención Básica. Método: Se trata de un estudio descriptivo con abordaje cualitativo realizado con 15 Enfermeros de las Estrategias de Salud de la Familia del municipio de Cajazeiras, Paraíba. Se utilizó para recolección de datos una entrevista semiestructurada, que ocurrió entre los meses de enero y febrero de 2016, tras la aprobación del Comité de Ética e Investigación. Resultados: Se percibió la existencia de algunos factores que perjudican la utilización de las tecnologías ligeras del cuidado, entre ellos, el tiempo reducido para realizar la atención a la población, estructura física inadecuada, inmediatez de la atención, la presión de la demanda para el trabajo. Conclusión: Es imprescindible que exista el apoyo de la gestión ofreciendo acciones de educación permanente para con los profesionales de la salud sobre la importancia de la utilización de las tecnologías interaccionistas en sus cotidianos de prácticas.

Palabras-clave: Atención de Enfermería. Enfermería. Estrategia de salud familiar.

\section{INTRODUÇÃO}

A dinâmica do processo de trabalho do enfermeiro é caracterizada pela diversidade de ações que o mesmo pode realizar, principalmente por atuar em áreas diversas, como na assistência, gerenciamento, pesquisa, ensino e nas questões políticas dessa categoria, bem como nos mais diversos assuntos que envolvem a área da saúde.

O cuidado é fundamental no processo de trabalho dessa categoria, pois envolve contato interpessoal com o sujeito e suas necessidades de saúde. Esse cuidado é determinante na assistência ao ser humano em suas necessidades e abrange comportamentos, atitudes e atos que se relacionam ao contexto social do usuário. Além disso, o cuidar, à luz da sensibilidade na enfermagem, compreende ações de atenção que perpassam o modelo biomédico. Está pautado em atividades subjetivas, como: contato; olhar nos olhos, além da percepção de aflições e sentimentos. ${ }^{1,2,3}$

Ao passo que o usuário é visto como ser humano, sujeito e ator social, se constrói, junto ao profissional, um cenário mais favorável à prestação de cuidados. Isso faz do cuidado de enfermagem uma prática social que se caracteriza por ação transversal, ou seja, presente em todos os cenários de atenção. Contudo, vale salientar sua relevância na Atenção Básica $(\mathrm{AB})$, visto por ser a principal porta de entrada para o Sistema Único de Saúde (SUS) e, por muitas vezes, o cuidado ao usuário neste campo de atenção determina a assiduidade do mesmo aos serviços, bem como o fortalecimento do autocuidado. ${ }^{4}$

As formas de organização das ações de saúde têm sido debatidas. De um lado, o entendimento conceitual da expressão "modelo assistencial" e a prática de 
atividades focadas apenas na doença. De outro, as características do que poderia ser um modelo orientado pelos princípios e diretrizes do SUS, mais abrangente e pautando-se na subjetividade do usuário, utilização de tecnologias leves e continuidade do cuidado. Apesar dos esforços existentes para o fomento de ações cada vez mais humanizadas na assistência à saúde, encontra-se, frequentemente, a recorrência de práticas baseadas no modelo de atenção tradicional, pautado em ações prescritivas e objetivas. ${ }^{5}$

$\mathrm{Na}$ prática do cuidado de enfermagem, o profissional utiliza estas tecnologias aliadas às práticas do cuidado que embasam a profissão. Dessa forma, o cuidado é visto como inerente ao ser humano, levando em consideração suas necessidades, o que $\mathrm{o}$ auxilia no enfrentamento às dificuldades da enfermidade para então promover a sua saúde. ${ }^{6}$ Uma alternativa para reformular o modelo tradicional de assistência é a utilização de tecnologias leves. As tecnologias leves são utilizadas nas relações humanas, como a produção de vínculo, autonomização, acolhimento, no encontro entre o profissional e o usuário. É por meio destas tecnologias que se constrói e se consolida a positividade entre os sujeitos envolvidos neste processo, como também a satisfação das necessidades dos indivíduos e a valorização (trabalhadores e usuários) como artifícios para intervirem na concretização do cuidado. ${ }^{7,8}$

Para tanto, o estudo baseou-se na seguinte questão norteadora: quais são os fatores dificultadores na realização das tecnologias leves no cuidado do enfermeiro na Atenção Básica?

Este estudo torna-se relevante para a enfermagem visto a necessidade de enfatizar os aspectos que possam dificultar a prática das tecnologias leves do cuidado, para evidenciar a necessidade de reconstrução do modelo assistencial, visando a formação do vínculo entre o profissional e o usuário, além da autonomização do mesmo e consequentemente a melhoria da qualidade dos serviços prestados. Dessa forma, objetivou-se identificar os obstáculos encontrados na execução de atividades relacionadas às tecnologias leves voltadas ao cuidado do enfermeiro na Atenção Básica.

\section{MÉTODO}

A pesquisa em questão é de natureza descritiva com abordagem qualitativa. Foi realizada na Atenção Básica do município de Cajazeiras, no estado da Paraíba. Essa cidade faz parte da 
$4^{\text {a }}$ Macrorregião de Saúde e da $9^{\mathrm{a}}$ Gerência Regional de Saúde da Paraíba. Nela, constam, atualmente, cadastradas 19 Unidades de Saúde da Família.

A pesquisa teve um número de 15 enfermeiros participantes que compõem um total de 23 Equipes de Saúde da Família, lotadas nas 19 Unidades da Atenção Básica de Cajazeiras. Trabalhar há mais de seis meses como enfermeiro na Atenção Básica foi o critério de inclusão adotado, entendendo-se esse período como mínimo para desenvolver familiaridade com a dinâmica desse cenário de atenção. Foram adotados como critérios para exclusão, estar de férias, licença-saúde ou afastado.

Utilizou-se a entrevista semiestruturada, sendo que as mesmas aconteceram entre os meses de janeiro e fevereiro de 2016, em local reservado nas Unidades Básicas de Saúde, em que estavam lotados os enfermeiros. Como processo metodológico, para análise dos dados recorreu-se ao Discurso do Sujeito Coletivo (DSC), que é um método que favorece a representação do pensamento de uma determinada coletividade.

Trata-se de uma estratégia metodológica de organização e tabulação dos dados qualitativos de natureza verbal, obtidos de depoimentos. Basicamente, consiste em analisar o material verbal coletado para se extrair dele as Ideias Centrais (IC) e suas correspondentes Expressões-Chaves $\quad(\mathrm{ECH}) .{ }^{9} \quad$ Esses depoimentos compõe a matéria-prima, sob a forma de um ou vários discursos-sínteses na primeira pessoa do singular, ou melhor, na primeira pessoa (coletiva) do singular, uma vez que ao mesmo tempo em que evidencia a presença de um ser individual do discurso, faz uma referência coletiva, pois esse ser individual fala em nome de uma coletividade.

A pesquisa iniciou-se a partir da aprovação do projeto pelo Comitê de Ética em Pesquisa (CEP) da Universidade Federal de Campina Grande, campus Cajazeiras sob o número do processo 1.347.458. A participação no estudo teve início mediante assinatura do Termo de Consentimento Livre e Esclarecido (TCLE), elaborado em duas vias, assinado pelo participante da investigação, bem como pelo pesquisador responsável. Em ambas as vias, constaram o contato telefônico dos responsáveis pela pesquisa e do CEP. Os componentes éticos e legais estão presentes em todas as fases da investigação, em conformidade com a Resolução 466/12 do Conselho Nacional de Saúde. 


\section{RESULTADOS}

No que se refere às atividades realizadas pelo enfermeiro e sua equipe junto à comunidade, percebeu-se a existência de alguns fatores que prejudicam a utilização das tecnologias leves do cuidado. Identificou-se quatro categorias principais que serão expostas e analisadas com o seu respectivo DSC.

A primeira categoria foi construída a partir das entrevistas de oito enfermeiros, a mesma abordou as fragilidades de realizar o acolhimento no cenário da Atenção Básica, conforme demonstrado a seguir:

Categoria 01 - Acolhimento na Atenção Básica: resistências e dificuldades na sua implementação:

DSC01: Na Unidade de Saúde da Família não se tem uma boa acolhida tanto por parte dos profissionais, tanto por parte dos pacientes, muitas vezes por conta do estresse do dia a dia, pela falta de conhecimento dos profissionais, que nem todos são da área de saúde, ou uma falta de capacitação, de acolher melhor o paciente que chega. Existe práticas muito deficitária ainda, não é assim muito aplicada não, a Atenção Básica infelizmente é muito engessada ainda, o acolhimento é pouco visto. Não há uma boa acolhida, não há uma humanização, às vezes só uma escuta de uma informação melhor que deve ser dada e não, não existe essa acolhida, existe às vezes por parte de alguns e de outros não. O que também não é percebido pela comunidade essa lógica do acolhimento que reclama do profissional que por vezes deixa de fazer.

A segunda categoria apresenta as vulnerabilidades enfrentadas no processo de efetivação da escuta. Nessa categoria houve a participação de seis enfermeiros:

A Categoria 02 - Fragilidades vivenciadas para a implementação da escuta:

DSC02: Assim, eu pelo menos, nas minhas atividades, eu tento ouvir, eu tento fazer o exercício de escutar, seja lá em que for, mas a população geralmente ela tá muito ligada à questão médica, aquele eterno desafio pra a enfermagem, acho que pra saúde de uma forma em geral, população tá muita ainda fixada no atendimento médico, na consulta, na medicalização, é muito voltado, ela ainda é muito presa ao método anterior, ao sistema de saúde anterior, então, tudo pra ele é uma urgência, não dá tempo esperar aqui pra gente atender, e não tão acostumado a uma consulta que tem escuta, né? Então, por muitas vezes, por mais que você diga, por mais que você mostre o quanto é importante você trabalhar a escuta qualificada, têm as fragilidades, que nem todo mundo tem a capacidade de se colocar na situação do outro, aí às vezes, não se coloca na situação, aí fragiliza o cuidado também dentro da equipe.

Autonomização é o tema referente a terceira categoria, sendo que cinco enfermeiros participaram da mesma.

Categoria 03 - Autonomização: saberes incipientes e ênfase em práticas ambulatoriais

DSC03: Se autonomização que eu tiver pensando for dá autonomia e empoderamento a 
esse paciente, eu acho que a gente tenta, mas como eu disse não há nenhum embasamento cientifico, tem as fragilidades, eu acredito que seja algo realmente ainda em construção ou a se construir, até a própria, assim, o fato de eles se sentirem livres a participar, né? Acho que realmente da minha prática diária é algo a ser construído, a se construir, a ser também aprimorado, porque ainda é algo muito, ainda é muito difícil, quando a gente fala em empoderamento é muito difícil, porque quem trabalha no PSF, e quem já passou por PSF, a gente vê que muitas vezes a gente tem que ofertar algo que tá totalmente fora do contexto pra conseguir trazer esse paciente. Até porque nós profissionais a gente adotou muito essa prática de fazer ambulatório, e a gente muito dentro da Unidade, muito dentro do PSF, e a gente não procurar sair, ultrapassar esse limite e sair, e buscar trazer esse usuário pra gente dessa forma. Além disso, quando ele busca o serviço e não dispõe do serviço, o usuário se sente inferior, acha que tá bolando de lugar pro outro, acha que o serviço ainda é insuficiente, também têm aqueles, que eles têm certa dificuldade em se sentir corresponsável, ele quer jogar a responsabilidade na equipe, na gestão, em todo mundo, ele nunca é responsável por nada, é, a gente tem dificuldade de trabalhar isso.

Por fim, a quarta categoria aborda sobre a resistência que a população demonstra quando o enfermeiro tenta utilizar das tecnologias do cuidado, na qual participaram sete enfermeiros.

Categoria 04 - Tecnologias do cuidado: resistência da população na sua implementação.
DSC04: Outro fator dificultante é a própria população, a questão da escuta, do vínculo, da forma como eu penso, eu acredito que pra população a questão como o empoderamento, não são todos que conseguem se empoderar realmente disso. Então, eu acho que a própria população ainda tem a mente um pouco fechada pra essas mudanças na atenção à saúde. O principal é o não entendimento da população, a não compreensão da população da importância do acolhimento e do vínculo, muitas vezes a gente faz uma consulta mais demorada porque precisa fazer uma escuta mais qualificada e o próximo reclama, entendeu. As pessoas não querem entender, algumas, assim, eles têm uma dificuldade em aceitar, né?

\section{DISCUSSÃO}

O DSC01 aponta o acolhimento como instrumento fundamental para $\mathrm{o}$ processo de trabalho bem-sucedido na $\mathrm{AB}$, todavia não há a implementação efetiva, sobretudo pela desarticulação entre os membros que compõem a equipe.

$$
\text { Estudo }^{10} \text { desenvolvido com }
$$
profissionais de saúde da $\mathrm{AB}$ de um município do interior do Estado do Rio Grande do Sul evidenciou que o acolhimento representa prática segmentada e não sistemática, de modo que os profissionais dos serviços acabam por implementá-lo, conforme compreensão individual, o que demonstra o desconhecimento da prática e o 
distanciamento da mesma dos princípios do SUS.

Muitos são os obstáculos que dificultam o desenvolvimento de prática acolhedora, se destacam, dentre esses, o tempo reduzido para realizar o atendimento à população, estrutura física inadequada, imediatismo da consulta e a pressão da demanda para o trabalho, que acarretam na insatisfação por parte dos profissionais e população. $^{11,12}$

Diante das dificuldades encontradas, deve haver o incentivo à implementação do acolhimento, objetivando utilizá-lo como ferramenta de comunicação entre os profissionais de saúde e a população, visando, principalmente, a melhoria da assistência e a resolubilidade dos problemas identificados.

Além disso, é perceptível ainda de acordo com o DSC01, a transferência de responsabilidade da não efetividade do acolhimento para a comunidade, quando o que acontece, muitas vezes, é a falta de sensibilização da população para tal prática. É de suma importância a utilização de ações educativas a fim de sensibilizar a comunidade acerca da relevância das tecnologias interacionistas, podendo contribuir para a maior aceitação das mesmas, visto que o acolhimento auxilia na busca por uma assistência longitudinal e organização da demanda do serviço, além de fomentar a acessibilidade aos serviços de saúde e integralidade do cuidado. ${ }^{13}$

No que diz respeito aos preparo dos profissionais, a gestão deve incentivar ações de educação permanente, bem como estratégias de utilização das tecnologias do cuidado nas práticas da AB. A fim de proporcionar maior aprimoramento científico e a adoção do modelo de saúde pautado na lógica da clínica ampliada, pois se verifica no discurso dos participantes deste estudo divergências entre conceituação e prática do acolhimento, tanto sobre a ótica do profissional, como pela dos usuários (na perspectiva dos enfermeiros), o que é um reflexo da fragilidade da formatação da equipe e das atividades que são ofertadas à população. ${ }^{14}$ De acordo com o DSC02, o exercício da escuta ativa por parte dos profissionais na $\mathrm{AB}$ é fragilizado, pois a população ainda se encontra resistente a essa "reformulação" do atendimento e demonstram preferência pelo atendimento médico, alem de ações centradas em prescrições medicamentosas. Isso pode ser consequência do processo de construção histórica dos modelos de atenção à saúde, sobretudo do modelo biomédico, centrado na hegemonia do profissional médico e na 
clínica com ênfase na doença, que ainda é muito presente nos serviços de saúde. ${ }^{15}$

Diante disso, esse paradigma assistencial influencia, tanto usuários da $\mathrm{AB}$, como os próprios profissionais de saúde que na execução de seu processo de trabalho não mostram à população alternativas de produção do cuidado além da consulta médica, o que resulta em ações individualistas aos sujeitos com agravos instalados. ${ }^{16,17}$

Destaca-se no discurso dos enfermeiros como um dos fatores dificultadores para implementação da escuta, a questão da procura por atendimento imediato na Estratégia Saúde da Família (ESF), decorrentes da fragilidade na orientação e informação da população para a utilização adequada dos servidos da rede de saúde. Diante disso, o serviço deve se reorganizar, realizando planejamento e a execução de ações que informem e sensibilizem a população acerca da procura apropriada da assistência, além da importância da escuta, objetivando a melhoria do atendimento. ${ }^{18}$

Observa-se também que a sobrecarga de funções e a dependência representam empecilhos à realização dessas práticas de cuidado por meio da escuta, o excesso de burocracia e até o próprio enfermeiro ao tomar para sim uma grande parcela de papéis. Convergindo com esses achados, em pesquisa realizada com enfermeiros da $\mathrm{AB}$ na cidade de Maracanaú, Ceará, identificaram que por conta da grande demanda e o tempo escasso de cada atendimento as ações que se fundamentam na utilização de tecnologias leves do cuidado como acolhimento, escuta qualificada e vínculo passam a ser deixadas de lado. Atrelado a isso se observa a resistência advinda da comunidade, sobretudo quando se relacionam com ações de promoção de saúde e prevenção de doenças. ${ }^{19}$

Foi possível atentar, no DSC03 dos enfermeiros, que um entrave para a autonomização é a insuficiência do saber desses profissionais, os quais padecem de capacitação para fomentar o empoderamento das pessoas que frequentam a AB. Além disso, nota-se, por parte dos profissionais, a priorização de ações ambulatoriais, com o escopo de atingir metas, muitas vezes devido imposição da gestão, o que estimula o distanciamento com a filosofia desse cenário de atenção.

Notou-se, de acordo com estudos $^{20,21}$, uma tendência nos profissionais de saúde a desvalorizarem a construção de relação dialógica com os usuários, o que dificulta a autonomização. 
Utilizam de práticas ambulatoriais hegemonicamente para promover o cuidado, como na solicitação de exames e encaminhamentos para consultas especializadas, quando muitas vezes as necessidades poderiam ser atendidas na própria Unidade de Saúde, a partir de outras possibilidades cuidativas.

Tal conduta leva os usuários a qualificar positivamente as ações ambulatoriais, práticas pontuais e com eficácia em curto prazo, e a desvalorizar as atividades de promoção da saúde e prevenção de agravos. Essas condições de produção do cuidado fincadas no modelo hegemônico não geram meios para o estreitamento das relações entre profissionais da saúde e comunidade, bem como dificulta a capacidade desses sujeitos para compreenderem e atuarem sobre si mesmo e seus contexto de vida e saúde.

O cuidado com base na clínica ampliada deve atuar como ferramenta potencial para transformar contextos de vida, já que essa ação passa a ser desenvolvida a partir da realidade e necessidade locais. ${ }^{22}$ Com isso, representa um modelo diferenciado de prestar cuidados, resultando em mudanças na vida dos indivíduos e o rompimento, gradativo, do modelo de prestações de serviços de saúde de forma tradicional.
Acredita-se, assim, que a autonomização dos atores sociais só será alcançada a partir do momento que o enfermeiro perceber a importância desses no processo de produção do cuidado, isto é, por meio da utilização adequada dos dispositivos tecnológicos em conjunto com os usuários, deixando, com isso, de ser construído como prática unilateral pelo enfermeiro e passando a ser de responsabilidade mútua.

Conforme o DSC04 supracitado, nota-se a menção dos enfermeiros sobre a resistência da população, também abordada no DSC02, que esses possuem na implementação das tecnologias leves do cuidado na $\mathrm{AB}$. Falar que há resistência é sim um fato, porém o enfermeiro, em determinadas situações, transfere toda a "culpa" para o usuário, sendo que esse profissional é também responsável na criação de obstáculos na implementação das tecnologias leve como prática cotidiana nesse cenário de atuação.

Algumas dificuldades são enfrentadas pelo enfermeiro no que se refere à construção de atividades educativas, como as de promoção da saúde e prevenção de agravos, isso se dá pela cultura curativista que acompanha grande parte dos usuários dos serviços de saúde. ${ }^{23}$ 
Para que essas atividades sejam valorizadas e aceitas pelos usuários e equipe multidisciplinar, deve ser trabalhado os atos educativos que fomentem os saberes prévios, visem a prática libertadora, e envolva todos os participantes na construção dos saberes.

Os enfermeiros devem acreditar nessas transformações e nos benefícios para a saúde de toda a população, para que assim os usuários compreendam as orientações e possam atuar em conjunto com a equipe no planejamento de ações, sendo necessário que eles tenham entendimento efetivo sobre os saberes compartilhados, a fim de compreenderem melhor a finalidade da utilização das tecnologias leves do cuidado, e deixarem de focar na necessidade de reprodução de práticas centradas nas tecnologias duras. ${ }^{23}$

Nota-se, finalmente, que adoção de ações educativas oportuniza aos atores sociais o desenvolvimento de suas capacidades individuais e coletivas visando à melhoria na qualidade de vida e saúde na busca cada vez mais por autonomia desses sujeitos na condução de suas vidas. Desse modo, dentre as ações da $\mathrm{AB}$, emergem as práticas educativas como ferramenta primordial para sensibilizar e transformar a visão dos profissionais e usuários sobre os objetivos desse espaço de atuação, e muito mais do que isso, a promover reflexões que conduzam modificações nas atitudes e condutas desses sujeitos.

\section{CONSIDERAÇÕES FINAIS}

No decorrer deste estudo, identificou-se os principais fatores que dificultam a implementação das tecnologias leves do cuidado como acolhimento, escuta, vínculo e empoderamento dos sujeitos atendidos na $\mathrm{AB}$ pelo enfermeiro.

$\mathrm{O}$ desconhecimento advindo tanto da população quanto dos profissionais dificulta o progresso do cuidado continuado, da formação do vínculo e no fortalecimento do uso das tecnologias leves. $\mathrm{O}$ acolhimento deve atender às singularidades dos sujeitos acolhidos na $\mathrm{AB}$, considerando seus valores sociais, condições socioeconômicas, culturais, psicológicas e espirituais.

No entanto, para que isso aconteça é necessário estruturar o trabalho da equipe, principalmente por meio de capacitações permanentes, de forma a garantir a inserção do usuário e proporcionar trabalho fundamentado na integralidade, considerando as necessidades, os determinantes e condicionantes de saúde da população, fortalecendo o vínculo e contribuindo para 
a existência de trabalho em equipe, que pressupõe mudanças nas relações interpessoais de trabalho e social.

Destaca-se, também, que os resultados obtidos nesta investigação possuem limitações, já que foi desenvolvido na $\mathrm{AB}$ de um município, de forma que não se pode generalizar as suas considerações para todos os demais serviços de abrangência nacional, uma vez que depende do contexto de saúde de cada população e da dinâmica do processo de trabalho dos enfermeiros de cada realidade.

Acredita-se que, ao revelar as particularidades e fragilidades que perpassam na implementação das tecnologias leves do cuidado, viabilizou-se melhor compreensão acerca da realidade do processo de trabalho do enfermeiro no âmbito da $A B$, o que poderá permitir cada vez mais a melhoria na condução das ações nesse espaço de atuação.

Finalmente, existe a necessidade de novas pesquisas, principalmente as de cunho intervencionistas, como também apoio da gestão oferecendo ações de educação permanente para com os profissionais da saúde sobre a importância da utilização das tecnologias interacionistas em seus cotidianos de práticas na $\mathrm{AB}$, visando, assim, transformações nesse cenário de atuação.

\section{REFERÊNCIAS}

1. Waldow VR, Borges RF. Caring and humanization: relationships and meanings. Acta Paul Enferm. [Internet]. 2011 [citado em 17 fev 2018]; 24(3); 414-8. Disponível em:

http://www2.unifesp.br/acta/pdf/v24/n3/v2 4n3a17_en.pdf

2. Queiroz BFB, Garanhani ML. Construindo significados do cuidado de enfermagem no processo de formação: uma pesquisa fenomenológica. Ciênc Cuid Saúde. [Internet]. out-dez 2012 [citado em 27 nov 2017]; 11(4):775-83. Disponível em:

http://ojs.uem.br/ojs/index.php/CiencCuidS aude/article/viewFile/21660/pdf

3. Souza MGD, Mandu ENT, Elias AN.

Perceptions of nurses regarding their work in the family health strategy. Texto \& Contexto Enferm. [Internet]. jul-set 2013 [citado em 05 out 2017]; 22(3):772-79. Disponível em: http://www.scielo.br/pdf/tce/v22n3/v22n3a25. pdf

4. Backes DS, Backes MS, Erdmann AL, Büscher A. O papel profissional do enfermeiro no Sistema Único de Saúde: da saúde comunitária à estratégia de saúde da família. Ciênc Saúde Colet. [Internet]. 2012 [citado em 13 jan 2018]; 17(1):22330. Disponível em: http://www.scielo.br/pdf/csc/v17n1/a24v17 n1.pdf

5. Fertonani HP, Pires DEP, Biff D, Scherer MDDA. The health care model: concepts and challenges for primary health care in Brazil. Ciênc Saúde Colet. [Internet]. 2015 [citado em 27 nov 2018]; 20(6):1869-78. Disponível em: http://www.scielo.br/pdf/csc/v20n6/en_141 3-8123-csc-20-06-1869.pdf

6. Silva RC, Ferreira MA. The practice of intensive care nursing: alliance among technique, technology and humanization. Rev Esc Enferm USP [Internet]. 2013 [citado em 18 fev 2018]; 47(6):1324-31. Disponível em: 
http://www.scielo.br/pdf/reeusp/v47n6/en_ 0080-6234-reeusp-47-6-01325.pdf

7. Thofehrn MB, Montesinos MJL, Arrieira IC, Àvila VC, Vasques TCS, Farias ID. Processo de trabalho dos enfermeiros de um hospital da Espanha: ênfase nas tecnologias de cuidado. Cogitare Enferm. [Internet]. 2014 [citado em 15 jan 2018]; 19(1): 141-46. Disponível em: http://www.redalyc.org/html/4836/483647 660021_2/

8. Merhy EE, Franco TB. Por uma composição técnica do trabalho em saúde centrada no campo relacional e nas tecnologias leves. Apontando mudanças para os modelos tecno-assistenciais. Saúde Debate [Internet]. set-dez 2003 [citado em 17 mar 2018]; 27(65):316-23. Disponível em:

https://www.arca.fiocruz.br/bitstream/icict/ 712/3/Travassos_Viacava_Landmann_Alo cacao\%20equitativa_2003.pdf\#page $=141$

9. Lefèvre F, Lefèvre AMC. O discurso do sujeito coletivo: um novo enfoque em pesquisa qualitativa (desdobramentos). Caxias do Sul, RS: EDUCS; 2003.

10. Borges JDM, Silva LAAO.

Acolhimento na atenção básica à saúde: saberes e práticas. Rev Enferm UFPE on line [Internet]. maio 2015 [citado em 16 mar 2018]; 9(5):7887-94. Disponível em: https://periodicos.ufpe.br/revistas/revistaen fermagem/article/view/10538/11443 11. Santana JCB, Fortes NM, Andrade AV, Soares APF, Lima JRM. Acolhimento em um serviço da atenção básica à saúde de Minas Gerais. Rev Enferm Cent-Oeste Min. [Internet]. mai-ago 2012 [citado em 21 fev 2018]; 2(2):166-76. Disponível em: http://www.seer.ufsj.edu.br/index.php/reco $\mathrm{m} /$ article/view/199

12. Nascimento MVN, Leite CCA, Rodrigues BS, Santos IA, Oliveira JOD, Bitú LSL. Integrated practices in health: a community therapy experience in basic care. Rev Enferm UFPE on line [Internet]. oct 2012 [citado em $10 \mathrm{fev}$ 2018]; 6(10):2586-95. Disponível em: https://periodicos.ufpe.br/revistas/revistaenf ermagem/article/view/7506

13. Penna CMM, Faria RSR, Rezende GP. Acolhimento: triagem ou estratégia para universalidade do acesso na atenção à saúde? Rev Min Enferm. [Internet]. 2014 [citado em 11 fev 2018]; 18(4):815-29. Disponível em: http://www.reme.org.br/artigo/detalhes/965 14. Kessler M, Lima SBS, Weiller TH, Lopes LFD, Ferraz L, Thumé E. Longitudinality in Primary Health Care: a comparison between care models. Rev Bras Enferm. [Internet]. maio 2018 [citado em 16 out 2018]; 71(3):1063-71.

Disponível em: http://www.scielo.br/pdf/reben/v71n3/0034 -7167-reben-71-03-1063.pdf

15. Santos AR, Carvalo MF, Santos RMM, Anjos SDS, Andrade CS. A produção do cuidado na atenção primária à saúde: uma compreensão teórico-filosófica. Ciênc Cuid Saúde. [Internet]. jul-set 2018 [citado em 27 out 2018]; 17(3). Disponível em: http://ojs.uem.br/ojs/index.php/CiencCuidS aude/article/view/39945/751375138365 16. Engela MHT, Rodarte AC, Rotondaro Júnior A, Seixas CT, Viegas SMF, Lanza FM. Uso das tecnologias em saúde na atenção básica às pessoas em condições de hipertensão arterial sistêmica. Rev Pesqui Cuid Fundam. [Internet]. jan/mar 2018 [citado em 15 nov 2018]; 10(1):75-84. Disponível em:

http://www.seer.unirio.br/index.php/cuidad ofundamental/article/view/5979/pdf_1

17. Pegoraro RF, Bastos LSN.

Experiências de acolhimento segundo profissionais de um centro de atenção psicossocial. Rev Enferm Atenção Saúde [Internet]. jan-jun 2017 [citado em 17 abr 2018]; 6(1):3-17. Disponível em: http://seer.uftm.edu.br/revistaeletronica/ind ex.php/enfer/article/view/1525

18. Puccini PT, Cornetta VK, Sahyom TZ, Fuentes ICP, Botta LMG, Puccini RF. Concepção de profissionais de saúde sobre o papel das unidades básicas nas redes de atenção do SUS/Brasil. Ciênc Saúde Colet. 
[Internet]. 2012 [citado em 19 mar 2018]; 17(11):2941-52. Disponível em: http://www.scielo.br/pdf/csc/v17n11/v17n 11a10.pdf

19. Fernandes MC, Silva LMS, Moreira TMM, Silva MRF. Factors involved in the management of nursing care: a descriptive study. Online Braz J Nurs. [Internet]. 2013 [citado em 17 ago 2018]; 12(3):522-33. Disponível em: http://www.redalyc.org/pdf/3614/3614339 17011.pdf

20. Lima LL, Moreira TMM, Jorge MSB. Produção do cuidado a pessoas com hipertensão arterial: acolhimento, vínculo e corresponsabilização. Rev Bras Enferm. [Internet]. 2013 [citado em 15 jun 2018]; 66(4): 514-22.

Disponível em:

http://www.scielo.br/pdf/reben/v66n4/v66n 4a08.pdf

21. Santos WJD, Giacomin KC, Firmo JOA. Avaliação da tecnologia das relações de cuidado nos services em saúde: percepção dos idosos inseridos na Estratégia Saúde da Família em Bambuí, Brasil.

Ciênc Saúde Colet. [Internet]. 2014 [citado em 13 fev 2018]; 19(8):3441-50.

Disponível em:

http://www.scielo.br/pdf/csc/v19n8/14138123-csc-19-08-03441.pdf

22. Feuerwerker LCM. Micropolítica e saúde: produção do cuidado, gestão e formação [Internet]. Porto Alegre: Rede UNIDA; 2014 [citado em 09 ago 2018]. Disponível em:

http://www.saude.sp.gov.br/resources/hum anizacao/biblioteca/dissertacoes-eteses/micropolitica_e_saude_laura_camarg o.pdf

23. Roecker S, Budó MDLD, Marcon SS. The educational work of nurses in the Family Health Strategy: difficulties and perspectives on change. Rev Esc Enferm USP. [Internet]. 2012 [citado em 17 ago 2018]; 46(3):641-49. Disponível em: http://www.scielo.br/pdf/reeusp/v46n3/en_ 16.pdf

RECEBIDO: $17 / 07 / 2018$

APROVADO: $12 / 11 / 2018$

PUBLICADO: $12 / 2018$ 